\title{
Lifecycle Management on Industry-University-Research Synergistic Innovation Mechanism
}

\author{
Ling Shen \\ Wuhan Donghu University,Jiangxia, Wuhan,Hubei, PRC \\ aleenapple@163.com
}

Keywords: Industry-University-Research; Synergistic Innovation; Lifecycle Management

\begin{abstract}
With the advent of science and technology, the enterprises rely heavily on the university and research. Industry, university and research are amalgamation together. The synergistic innovation mechanism has been preliminarily established. The mechanism, however, ignore the whole-process management. Since multi parties engaged in the project, a series of rules should be setup for the Synergistic Innovation Mechanism. In this paper, we employ lifecycle management which is a practical and systematic framework in software engineering in synergistic innovation mechanism. A series of approaches has been applied in the mechanism. Empirical study in Wuhan Donghu University demonstrates the effectiveness of the proposed methods.
\end{abstract}

\section{Introduction}

With the development of global economy and industry, the amalgamation of industry, university and research became to be the new research focus [1-4], and the new research on synergistic innovation mechanism began spring up. In general, the collaboration of industry-university-research (IUR for short) didn't have a fixed pattern. Oversea companies have more experiences in industry-university-research collaboration. For example, there are large amounts of high-tech companies such as Google, Facebook, and CISCO gave birth in campus in Silicon Valley. For the above reasons, the industry and university are interactive naturally in US. The IUR relation, however, is separated from each other in China. The Chinese high-tech companies normally didn't grow from the campus. Therefore, many studies have been devoted in establishing the relation of IUR. These works, however, are very complicated since different patterns should be adopted according to the actual situation.

Synergistic Innovation refers to a qualitative change in the efficiency generated by the participating factors through the interaction mechanism based on their individual efficiency improvement in the process to play their respective roles. Research on the enterprise innovation often follows a technology roadmap, which guide the enterprise from the single innovation to collaboration innovation. The Synergistic Innovation Mechanism of IUR includes a series of stage, which incur multiple parties to engage in.

Then how to settle the problem becomes an important problem. In this paper, we employ an important concept: lifecycle management, which is from software engineering to design a technology roadmap. In this roadmap, we investigate the theory of construction, management, operation and benefit distribution in the collaboration of IUR. Our approaches take advantage of software engineering into synergistic innovation mechanism for the first time. Important policy and rules have been setup to aid the domestic industry. It will help the development of both the industry and the university.

Finally, empirical studies have been conducted on the Wuhan Donghu University. Several famous enterprises have collaborated with the university. On one hand, the university invests its intelligence superiority to help enterprises in product research and development. The industry get large amount of benefits in the collaboration. On the other hand, the enterprise brings its experiences to the university. The two-party establish joint laboratories and training bases. The experts and engineers in the training bases can lead the research group, and teach the students. 
The rest of this paper is listed as follows. Section 2 investigates the industry-university-research synergistic innovation mechanism. Section 3 depicts the lifecycle management in software engineering and how to transform synergistic innovation mechanism into a lifecycle management. Empirical studies have been conducted on Section 4. Comparative experiments demonstrate the effectiveness of proposed method. Section 5 is the conclusion of the paper.

\section{Industry-University-Research Synergistic Innovation Mechanism}

Synergistic innovation refers to a qualitative change in the efficiency generated by the participating factors through the interaction mechanism. Our synergistic innovation has several characteristics of synergistic innovation. The first characteristic is that synergistic innovation constitutes the industry, university and research [5]. The multi-party has complicated relation which makes the problem difficult to solve. A framework has been proposed to establish a supply and demand network with multi-functional was developed. It is composed by the "supply-demand flows" among enterprises. As far as we can see, communication is the main methods for enterprises to collaborate, and resource sharing is the basic ensure of enterprise collaboration. All the goal of collaboration innovation is to enhance the compatibility of the alliance with external companies. So we should establish an all-round collaboration in the mechanism. In this mechanism, the multi-party should be stakeholders. The industry, university and research will participate in a Win-win strategy. They will benefit from the collaboration. In the end, the collaboration will step into a beneficial economic cycle. The mechanism, however, is very ideal. Since different situation may occur, we should design an overall consideration. In our plan, not only successful collaboration but also failing collaboration will be designed. Stop loss and exit plan should be prepared before the collaboration.

\section{Lifecycle Management Method}

In this section, we firstly introduce the lifecycle management concept in software engineering. Then we employ the method into industry-university-research collaboration.

\subsection{Lifecycle Management Concept and Waterfall Method}

Lifecycle management is an important concept used in software engineering. It describes a process for planning, creating, testing, deploying, and maintain an information system. Lifecycle management is composed of a number of clearly defined and distinct work phrases which are used by system engineers and developers to plan for, design, build, test, and deliver information systems. Computer systems are complex and often link multiple traditional systems potentially supplied by different software venders. To manage this level of complexity, a number of models or methodologies have been created, such as "waterfall” [6], "spiral”, etc. The waterfall model is a sequential design process, in which process is seen as flowing steadily downwards (like a waterfall) through the phrases of conception, initiation, analysis, design, construction, testing, production and maintenance.

\subsection{Lifecycle Management in Collaboration}

In our setting, the industry-university-research collaboration is also a complicated process, in which multi-parties engaged in. This observation inspired us to transform the collaboration process into a lifecycle management. We divide the overall-process into four stages, which are construction, management, performance, and benefit distribution of the collaborative activities. The four stages are shown in following.

1) Construction Stage

a. Collaboration Motivation Mechanism

This step means the motivation to carry collaboration from the industry, university and research institute. The requirements mainly come from the market, technology innovation and the government.

b. Collaboration Entrance Mechanism

In this step, industry and enterprise choose each other, to form an organization. Two conditions should be met. One condition is common goal. The other is resource complementarity. 
c. Collaboration Quit Mechanism

While the internal or external circumstance changed, collaboration carries out self-renewal. In general, there are two cases: the member quit initiatively or is obliged to quit according to the contract. In this step, it is important to establish a fair and justice access mechanism to ensure the quit and compensation of the members.

2) Management Stage

a. Collaboration Negotiation Mechanism

Effective means should be taken to coordinate the benefits of different parties.

b. Collaboration Trust Mechanism

In this step, we should setup a trustful platform, in which the industry and university could exchange their innovative fruits.

3) Performance Stage

a. Resources Integration Mechanism

The resources scattered everywhere should be integrated to a serve the collaboration. The resources include: technology information, large-scale instrument and equipment, laboratory, Intermediary service mechanism.

b. Cooperation Mechanism

In this step, productivity factors and knowledge have been deployed in the chain. Every node should abide by the rules.

c. Achievement Input Mechanism

The achievement should be input into the collaboration. Method, scheme, and control method has been established. Scientific achievement should be adjusted to fit the collaboration.

4) Benefits distribution Stage

a. Risk Sharing Mechanism

The collaboration members should share the project risk. The more risk one carry, the more benefits he should get. The other is risk pre-warning mechanism.

b. Monitoring Mechanism

A monitoring mechanism should be built, to prevent the risk and the loss.

\section{Empirical studies}

\subsection{Investigation Setup}

In order to evaluate the proposed method, we collect empirical data to validate our approaches. The experiments are chosen in Wuhan Donghu University, which is a private university in Hubei, China. In 2014, the university establishes relationship with two famous high-tech corporations: Neusoft and Pectera. Nuesoft is a leading IT solution and service provider, while Pectera is the largest software outsourcing company. The university and the two companies form collaboration. Two laboratories have been built. Teachers and students go the innovation lab, and become designer and developers. They acquire true experience within the project. On the other hand, many works have done by the students. The enterprises also acquire the profit.

\subsection{Result}

In this section, we conduct questionnaire survey in our investigation. Both the university and the enterprise accept the survey. Totally 30 students and 3 teachers from the university and 3 staffs from enterprise received the questionnaire and returned it. In our experiment, about $83.6 \%$ students are satisfactory with the project. About $100 \%$ teachers give A score or above to the project. For the staffs in enterprise, about $66.7 \%$ staffs give A score or above to the project. This result means that students are eager to engage in the training system of enterprise at present. On the other, majority of staffs give positive reviews to the project, while minor of staffs give negative reviews to the project. We should improve our framework to adapt the collaboration. 


\section{Conclusions}

In this paper, we investigate industry-university-research synergistic innovation mechanism. To handle the complication of the multi-party engaged collaboration, we transform the process into a lifecycle management. Waterfall method in software engineering has been utilized in collaboration. A series of approaches has been applied in the mechanism. Empirical study in Wuhan Donghu University demonstrates the effectiveness of the proposed methods.

\section{Acknowledgements}

This work was supported by the grants soft science project 2015BDH112, from science and technology office of Hubei Province.

\section{References}

[1] Fan, De Cheng, and X. X. Tang. "Performance evaluation of industry-university-research cooperative technological innovation based on fuzzy integral." International Conference on Management Science and Engineering IEEE, 2009:1789-1795.

[2] Cao Jing, Shen Ming, and Fan De-cheng. "The game analysis on interests distribution of industry-university-research cooperative technology innovation." (2011):1081-1087.

[3] Shen, Ying, L. J. Zhang, and X. L. Guo. "Study on the industry-university-research cooperation system: From the angle of labor division and cooperation." Industrial Engineering \& Engineering Management IEEE International Conferen (2010):1736 - 1740.

[4] Liang-Ping, Wang, and Y. Shan-Lin. "Study on the Dynamic Mechanism of the Universities' Independent Innovation Based on Industry-University-Research Institute Alliance." The International Conference on E-Business and E-Government, ICEE 2010, 7-9 May 2010, Guangzhou, China, Proceedings 2010:4628-4631.

[5] Chen, Li, and F. Xu. "Research on SDN Enterprise’s Collaboration Innovation Mechanism." Asian Social Science 7.1(2010).

[6] Royse, By W W. "Managing the development of large software systems." Proceedings IEEE Wescon 26(2010):328-338. 